\title{
Die Populismen der Lega Nord und der Wandel ihrer Volksvorstellungen
}

\author{
Giorgia Bulli
}

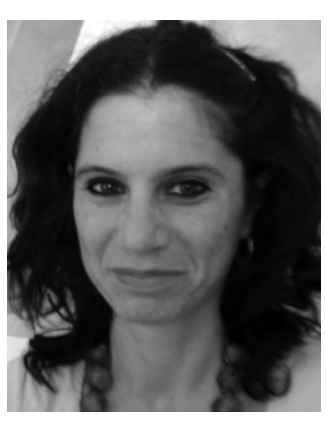

Dr. Giorgia Bulli, geboren 1974 in Florenz; aktuell Researcher an der Cesare Alfieri Universität Florenz. Zuvor Studium der Politikwissenschaften in Florenz und Heidelberg sowie Promotion in Politikwissenschaften an der Universität Florenz. Inhaberin der Lehrstühle „Politische Kommunikation" und „Politische Sprache“ an der politikwissenschaftlichen Fakultät der Universität Florenz.

\section{Abstract}

The polysemic nature of populism is perfectly reflected in the multifaceted populism of the Italian Lega Nord (LN). Born as the supposed receptacle of cultural and autonomist tendencies of the Northern regions, the party soon developed into a genuine right-wing populist party. Its programmatic platform and rhetoric - one of the major factors of its electoral success - reflect the breadth of the LN's appeal to the people. The party program and rhetoric constantly fluctuate between an identity-based and a protest-based call for a deep renovation of the relationship between the people and its élite. This renovation includes the call for more autonomy of the Northern Italian regions - ranging from federalism to secession - which coexists within the LN platform with more genuine stances of European right wing populist parties: Anti-establishment and anti-party positions, anti-immigration in addition to law and order issues, the need to return to traditional family values and the rejection of a multicultural society. In order to correctly interpret one of the most important political actors of the Italian party system of the last two decades, the article analyses these programmatic dimensions as they shift during the evolutionary phases of the LN. Despite the recent crisis which affected the party and its leadership, the LN represents a prototypical example of how the contradictions of liberal democracies, due to which populism prospers, have been successfully exploited.

\section{Einführung}

Im weiten Umfeld der rechtspopulistischen Parteien, ${ }^{1}$ die seit Anfang der 1980er Jahre in Westeuropa aufkommen, kann die Lega Nord (LN) sicherlich als ein prototypisches Beispiel betrachtet werden. ${ }^{2}$ Nicht selten jedoch wurde die LN als eine rechtsextreme Partei klassifiziert und unter die breite Gruppe rechts-

1 Für hilfreiche Kommentare und Korrekturen bin ich Paula Diehl sehr dankbar.

2 Vgl. Marco Tarchi, L'Italia populista. Dal qualunquismo ai girotondi, Mailand 2003, S. 135. 
extremistischer oder rechtsradikaler Parteien subsummiert. ${ }^{3}$ Verschiedene typische Elemente der Darstellung der politischen und sozialen Realität Italiens sowie der Art und Weise der Parteimobilisierung durch die LN sind für diese mehrdeutige Klassifizierung verantwortlich.

Um Unsicherheiten zu vermeiden, sei klargestellt: Die LN gehört nach Auffassung der Autorin zu jenen rechtspopulistischen Parteien, die einen Identitätsund Protestpopulismus nutzen um ihre Betätigung zu legitimieren und um dieser Betätigung strategisch den größtmöglichen Effekt zu geben, und zwar sowohl hinsichtlich der Wahlergebnisse als auch des Einflusses auf die öffentliche Meinung.

Es ist daher wichtig zu verstehen, wie die Partei im Laufe der Jahre die öffentliche Debatte beeinflusst hat - im Blick auf dominante Themen wie Einwanderung, die Rückkehr zu traditionellen Werten - wie etwa die Familie und den christlichen Ursprung westlicher Gesellschaften - der Skepsis gegenüber einer europäischen Integration oder einer steuerfeindlichen Politik. Die Partei, die von vielen auch als Vertreterin eines Ethnoregionalismus betrachtet wird, hat außerdem eine Diskussion um die föderalistische Verfasstheit des italienischen Staates vorangebracht.

Typisch für die rechtspopulistischen Strömungen Westeuropas ist ein Demokratiedefizit in der inneren Parteiführung und ein organisatorischer Aufbau, der in diesem Fall bis vor wenigen Monaten Umberto Bossis charismatische Führungsrolle in den Mittelpunkt stellte. Eine divergierende Interpretation programmatischer und organisatorischer Elemente der LN führt wahlweise zur Einordnung der Partei in den rechtsradikalen Bereich oder unter die Kategorie des Populismus. Im Besonderen geht es hier um die einwanderungsfeindliche Haltung, die radikale Ablehnung eines Multikulturalismus sowie eine gewisse Form von Antiparlamentarismus. Je nach Entwicklungsphase der Partei wurde zudem der Auftritt als parteienfeindliche Bewegung mit einer staats- bzw. systemfeindlichen Perspektive verbunden, was in den Medien bisweilen das öffentliche Bild einer subversiven Partei entstehen ließ. Es wird daher zu zeigen sein, wie dieses stark oppositionelle Image der LN die Möglichkeit eröffnet hat, sich als wahre Vermittlerin der Stimme der Bürger des Nordens zu gerieren. Im weiteren Verlauf wurde die Partei durch ihre Wahlerfolge in eine Phase der Institutionalisierung gedrängt, bald verbunden mit Regierungsbeteiligungen. Das Profil der Partei schwankte im Laufe der Zeit zwischen institutionellen und antipolitischen Perspektiven, getreu dem eigenen Motto „Lega di Lotta e di governo“(„Liga des Kampfes und der Regierung"). Daraus ergeben sich ähnliche Widersprüche wie bei anderen populistischen Parteien mit Regierungsbeteiligung. In diesen Widersprüchen wiederum manifestiert sich die ambivalente Beziehung zwischen Populismus und Demokratie - eine vernichtende populistische Kritik ist zugleich Pro-

3 Vgl. Paul Hainsworth, The Extreme Right in Western Europe, New York 2008; Herbert Kitschelt/Anthony Mac Gann, The Radical Right in Western Europe. A Comparative Analysis, Ann Arbor 1995. 
jektionsfläche für die Wünsche des gewöhnlichen Bürgers und beinhaltet auch ein Moment der demokratischen Erneuerung.

Dieser Beitrag dient folglich einer Beschreibung jener Eigenschaften, die es der LN ermöglicht haben, sich als eine der erfolgreichsten rechtspopulistischen Parteien Westeuropas durchzusetzen. Von besonderer Relevanz wird die Analyse jener Faktoren sein, die sich gewissermaßen auf halbem Wege zwischen Populismus und Rechtsradikalismus erfassen lassen und die ermöglicht haben, dass die LN sich im Laufe weniger Jahre als einer der wichtigsten Protagonisten im Parteienspektrum Italiens durchgesetzt hat. Die LN hat maßgeblich dazu beigetragen, dass der Ton in der Debatte um zentrale und problematische Themen, wie insbesondere die Einwanderungspolitik, verschärft wurde. Ein ständiger Begleiter dabei ist das typisch populistische Argument der Kluft zwischen Regierenden und Regierten. Gleichzeitig gelang es der Lega, die Aufmerksamkeit der politischen Akteure Italiens auf andere, für die öffentliche Meinung sensible, lange nicht beachtete Themen, wie die sogenannte „Nordfrage“ („questione settentrionale") zu lenken. ${ }^{4}$

Nachfolgend werden hier die Entwicklungsphasen der LN vom Ende der 1980er Jahre bis zur heutigen Krise zurückverfolgt, indem für jede Periode sowohl die populistischen Züge der Partei, als auch diejenigen Aspekte hervorgehoben werden, welche deutlich auf ein klassisch rechtsradikales Repertoire hinweisen. ${ }^{5}$ Eine Rekonstruktion der Parteientwicklung zeigt, dass die LN in der Lage war, typisch antipolitische Forderungen populistischer Parteien zu nutzen. Zugleich ist es ihr gelungen, auch als institutionell identifizierbarer Akteur wahrgenommen zu werden, wenngleich aus der Regierungsrolle auch Widersprüche resultieren. ${ }^{6}$ Aus dem breiten thematischen Spektrum der LN werden wir folgende analysieren, auch weil sie unter komparatistischen Gesichtspunkten am meisten einer populistischen Natur der Partei entsprechen: Parteienfeindlichkeit und Kritik gegenüber der repräsentativen Demokratie, Einwanderung, die

4 Vgl. Ilvo Diamanti, Il male del Nord. Lega, localismo, secessione, Rom 1996; Anna Cento Bull/Mark Gilbert, The Lega Nord and the Northern Question in Italian Politics, Houndsmills/New York 2001; Marga Gomez-Reino Cachafeiro, Ethnicity and Nationalism in Italian Politics. Inventing the Padania: the Nord and the Northern question, Burlington 2002; Roberto Biorcio, La rivincita del Nord. La Lega dalla contestazione al governo, Rom 2010.

5 Vgl. Biorcio, La rivincita del Nord, S. VIII.

6 Über die populistischen Parteien in der Regierungsrolle und die Unmöglichkeit, typische Versprechen einer Protest-Partei zu halten, siehe: Michael Minkenberg, The Radical Right in Public Office: Agenda Setting and Policy Effects. In: West European Politics, 24 (2001) 4, S. 1-21; Martin Schain/Aristide Zolberg/Patrick Hossay (Hg.), Shadows Over Europe: The Development and Impact of the Extreme Right in Western Europe, Houndsmill 2002; Reinhard Heinisch, Success in Opposition - Failure in Government: Explaining the Performance of Right-Wing Populist parties in Public Office. In: West European Politics, 26 (2003) 3, S. 91-130; Michelle Hale Williams, The impact of radical right wing parties in West European Democracies, New York 2006. 
Änderung der Gebietsordnung des italienischen Staates (vom Föderalismus zur Sezession), moralische Werte und schließlich die Familie.

Die Besetzung dieser Themenfelder verweist auf Gründe für den Erfolg der LN wie auch auf Gründe für eine Krise der Parteien in Italien. Hinsichtlich der Beziehung zum italienischen Parteiensystem kann man von einer konstanten Erneuerung bzw. einer chamäleonartigen Anpassung der Lega sprechen; hinzu kommen die wechselhaften Umstände des Wandels von der Ersten zur sogenannten Zweiten Republik innerhalb weniger Jahre. Während dieses Überganges, der für viele noch unvollendet oder sogar überholt ist, kam der Lega eine tragende Rolle zu. ${ }^{7}$ Bossis Partei ist es dabei tatsächlich gelungen, eine neue Art der Kommunikation einzuführen. Diese Kommunikation hat ihr Fundament in einer volkstümlichen Sprache mit vielen idiomatischen, aus dem Dialekt stammenden und oft auch vulgären Ausdrucksweisen. ${ }^{8}$ Jene neue Form der Volksnähe hat der populistischen Botschaft der LN schließlich Form und Substanz gegeben.

In den westeuropäischen Demokratien zeigt die von populistischen Parteien ausgeübte Kritik und die Betonung einer Kluft zwischen Regierenden und Regierten, wie problematisch die Beziehung zwischen sogenannten redemptive und pragmatic faces der Demokratie sein kann - Margaret Canovan hat dies sehr schlüssig dargelegt. ${ }^{9}$ Außerdem bringt diese Kritik auch das instabile Gleichgewicht zwischen konstitutionellen und ideologischen Fundierungen liberaler Massendemokratien zu Tage, wie die Analyse von Mény und Surel aufzeigt. ${ }^{10}$ Wie Weyland in seinen Studien zeigt und wie kürzlich auch Mudde und Kaltwasser unterstrichen haben, konzentrieren sich die im südamerikanischen Kontext entwickelten Reflexionen zum Populismus hingegen gerade auf dessen Fähigkeit, eine Gesellschaft über die Wahlen hinaus zu mobilisieren. ${ }^{11}$ Ziel dieses Kapitels ist es also, die ambivalente Beziehung zwischen Populismus und Demokratie zu

7 Vgl. Mauro Calise, La Terza Repubblica. Partiti contro presidenti, Rom 2006.

8 Über die Eigenschaften der politischen Sprache der LN siehe: Paolo Desideri, L'italiano della Lega/1. In: Italiano e oltre, 8 (1993), S. 281-285; Paolo Desideri, L'italiano della Lega/2. In: Italiano e oltre, 9 (1994), S. 22-28; Roberto Iacopini/Stefania Bianchi, La Lega ce l'ha crudo. Il linguaggio del Carroccio nei suoi slogan, comizi e manifesti, Mailand 1994; Lorella Cedroni, Il linguaggio politico della transizione. Tra populismo e anticultura, Rom 2010.

9 Vgl. Kay Lawson/Peter H. Merkl (Hg.), When parties fail. Emerging Alternative Organizations, Princeton 1998; Margaret Canovan, Trust the people! Populism and the two faces of democracy. In: Political Studies, 47 (1999), S. 2-16.

10 Vgl. Yves Mény/Yves Surel, Populismo e democrazia, Bologna 2001.

11 Vgl. Kurt Weyland, Clarifying a Contested Concept: Populism in the Study of Latin American Politics. In: Comparative Politics, 34 (2001) 1, S. 1-22; ders., Neopopulism and Neoliberalism in Latin America: Unexpected Affinities. In: Studies in Comparative International Development, (1996), S. 3-31; Cas Mudde/ Cristóbal Rovira Kaltwasser, Voices of the Peoples. Populism in Europe and Latin America Compared, Kellogg Institute Working Paper, Notre Dame 2011; Cristóbal Rovira Kaltwasser, Skizze einer vergleichenden Forschungsagenda zum Populismus. In: TD, 8 (2011) 2, S. 251-272. 
verdeutlichen und zu zeigen, welche Form diese Beziehung im Zusammenhang mit der Entwicklung einer der Parteien annimmt, welche die Umwandlung des italienischen Parteiensystems im Laufe der letzten Jahrzehnte beeinflusst hat.

\section{Vom Ende der 1980er Jahre bis zur Mitte der 1990er Jahre - ein Ursprung zwischen Regionalismus und Antipolitik}

Der Ursprung der Lega Nord ist das Ergebnis eines kulturellen und regionalistischen Phänomens, das Ende der 1980er Jahre zur Entstehung einer Gesamtheit kultureller Organisationen geführt hat, welche die Absicht hatten, der Sprache und der Tradition einiger Gebiete Norditaliens neuen Auftrieb zu geben. Die erste dieser Organisationen, die „Società Filologica Veneta“, wurde 1978 von Franco Rocchetta mit der Absicht gegründet, die venezianische Sprache und Kultur zu bewahren und zu verbreiten. Diese Organisation kam bald mit der von Bruno Saldavori geleiteten „Union Valdôtaine“ in Kontakt. Letztere war ihrerseits mit dem zukünftigen Kopf der LN in Kontakt, wobei Umberto Bossi zu jener Zeit noch nicht politisch aktiv war. Bossi, geboren in Varese, also in der Lombardei, gründete 1982 die „Lombardia Autonomista“, eine Zeitschrift, die ihren Fokus auf kulturelle und traditionelle Aspekte der Lombardei richtete. Nach kurzer Zeit wurden ähnliche Bewegungen auch im Aostatal, in Südtirol und im Friaul gegründet.

Die LN ist somit aus einem Vereinigungsprozess einiger der vielen autonomistischen Ligen entstanden, die sich seit Anfang der 1980er Jahre in Venezien, im Piemont, im Aostatal und in der Lombardei entwickelt hatten. Wie Biorcio allerdings erkennt, „ist das neue kollektive Subjekt nicht als einfache Aggregation individueller Akteure, die Interessen und gleichartige soziale Bedingungen teilten, entstanden“. ${ }^{12}$ Der gemeinsame Nenner der Ligen bestand über die Jahre in dem Bestreben, traditionelle und sprachliche Werte der nördlichen Regionen zu verteidigen. Über Sprachdifferenzen hinaus, die in anderen Beiträgen hinreichend dargestellt worden sind, ist diese Periode durch die Suche nach der Eigentümlichkeit des Lokalismus gekennzeichnet. Diese Suche nimmt die Form und Substanz einer Neubewertung sprachlicher und - in einem breiteren Sinne - kultureller Eigenart an.

Die geographische Verortung dieser neuen Bewegung, welche im ersten Moment eine im Wesentlichen kulturelle Gestalt annahm, verweist bereits auf die bald primäre Rolle des Nordens oder genauer des Nordostens. Innerhalb jener geographischen Zone verbreiteten sich die Keime der später sogenannten

12 Roberto Biorcio, La Padania promessa. La storia, le idee e la logica d'azione della Lega Nord, Mailand 1996, S. 16. 
„questione settentrionale“ („Nordfrage“). Dieses „Übel des Nordens“13 wird gebildet aus einer Mischung von Unzufriedenheit der über den Steuerdruck besorgten produktiven Schichten und der allgemeinen Wahrnehmung über eine mangelhafte und weit entfernte politische Vertretung. ${ }^{14}$ Nicht umsonst hat man die steuerkritische Argumentation der LN mit „einer aktualisierten Version des Poujadismus" 15 verglichen. Obgleich das Phänomen der Ligen erst Ende der 1980er Jahre politische Relevanz erreichte, waren die Voraussetzungen für die Entwicklung antizentralistischer und typischer Slogans der LN schon zum Anfang des Jahrzehnts vorhanden - „Roma ladrona“ ist ein Beispiel für solche Slogans.

Es existieren in der Literatur zum Thema zwei wichtige Vorfahren der LN, die Lega Veneta ${ }^{16}$ und die Union Valdôtaine. Die Liga Veneta, die schon seit Anfang der 1980er Jahre im Bereich der Neuentdeckung der territorialen und kulturellen Identität Veneziens aktiv war (dabei unterstützt von der Società Filologica Veneta), begann einen Teil der Wähler des PSI (Partito Socialista Italiano) und mehr noch der DC (Democrazia Cristiana) abzuschöpfen. Dies gelang ihr durch die Kommunikation einer Krise der weißen Subkultur nach den Säkularisierungsprozessen der venetischen Gesellschaft ab Anfang der 1960er Jahre. ${ }^{17}$ Bruno Salvadori, der politische Kopf der Union Valdôtaine, hat Umberto Bossis politisches Engagement entschieden beeinflusst. In diesem Zusammenhang ist es wichtig, auf das repräsentative Symbol der Lega Lombarda hinzuweisen: Ein Bild des Alberto da Giussano, jenes Heerführers, der im zwölften Jahrhundert die Carroccio-Gesellschaft gegen Kaiser Barbarossa mit der Absicht geleitet hatte, die zur Lega Lombarda zusammengeschlossenen vereinigten Gemeinden Norditaliens zu unterwerfen (Pontida-Schwur). ${ }^{18}$ Aus alledem wird ersichtlich, dass symbolische und mythische Komponenten eine sehr wichtige Rolle in der Identitätsbehauptung der Liga-Bewegung gespielt haben. Die Schaffung von Ritualen, insbesondere die Treffen auf der Wiese in Pontida, das Was-

13 Diamanti, Il male del Nord, 1996. Unter dem Ausdruck „Male del Nord“ versteht man das Gefühl der ökonomischen „relativen Deprivation“ der nördlichen italienischen Regionen, ein Punkt der von der LN mit Blick auf den römischen Zentralismus ausgenutzt worden ist.

14 Vgl. Ilvo Diamanti, Mappe dell'Italia politica, Bologna 2009, S. 69 f. Während dieser Periode sind die Stimmen für die Lega „unter den Angestellten und selbstständigen Arbeitern der kleinen Unternehmen " $\mathrm{zu}$ finden. Sie ist sowohl unter den am wenigsten praktizierenden Katholiken stark, als auch unter denjenigen, die für die DC gewählt hatten und für die anderen Regierungsparteien, ohne sich mit diesen zu identifizieren.

15 Tarchi, L'Italia populista, S. 146; Maria Antonietta Confalonieri, Identità, interessi e carisma nei movimenti populisti: la Lega Nord e il poujadismo. In: Quaderni di scienza politica, 4 (1997) 1, S. 53-84.

16 Vgl. Ilvo Diamanti, La Lega. Geografia, storia e sociologia di un soggetto politico, Rom 1993, S. 45.

17 Vgl. Diamanti, La Lega, S. $47 \mathrm{ff}$.

18 Vgl. Daniele Vimercati, I lombardi alla nuova crociata, Mailand 1990, S. 3-10. 
serholen am Fluss Po und andere Aktivitäten dienen der Aufwertung einer padanischen Identität. ${ }^{19}$

Während der Einfluss der Säkularisierung am Anfang der „prähistorischen“ Periode der Lega als Grund des Erfolges der Autonomiebestrebungen erscheint und es vor allem die Lega Veneta war, welche die traditionellen Forderungen vertrat, so übte später die Lega Lombarda unter der Leitung von Umberto Bossi einen immer größeren Einfluss aus. Dies gelang ihr auch dank der organisatorischen Bemühungen und der strategischen Strukturierung, die ihr Anführer der zunächst kleinen Gruppe von Mitgliedern und Sympathisanten auferlegte. ${ }^{20}$

Während der gesamten 1980er Jahre erreichten die Ligen nur bescheidene Wahlergebnisse: Ab 1989, mit der Gründung einer Allianz (Alleanza Nord) nahmen sie an den Europawahlen teil und erhielten italienweit 1,8 \% der Stimmen. Allerdings stießen sie auf große Resonanz in der Lombardei, wo die Liste $8 \%$ der Stimmen erhielt, mit den besten Ergebnissen in den Städten Varese, Sondrio, Como und Bergamo. Im selben Jahr wurde die LN offiziell aus einer Union mehrerer Ligen heraus gegründet, darunter die Lega Veneta, die Unione Ligure, die Lega Emiliana-Romagnola und natürlich die Lega Lombarda. Der Erfolg der Lega Nord stellte sich im folgenden Jahr während der Regionalwahlen ein, wo sie eine Plattform präsentierte, in der die Autonomiebestrebungen um eine Kritik des bestehenden Parteiensystems ergänzt wurden.

In seiner Analyse der Entwicklung der ideologischen Plattform der Lega hat Biorcio den Übergang von einem regionalistischen Populismus hin zu einem nordistischen Populismus herausgearbeitet. ${ }^{21}$ Diese Bezeichnung bringt zwei Schlüsselaspekte zum Verständnis des Lega-Phänomens zum Vorschein: den Populismus einerseits und die schon erwähnte „Nordfrage“ andererseits. Beide waren im Erbgut der Lega Autonomista Lombarda in nuce schon vorhanden, aber entwickelten sich im Laufe der 1990er Jahre sehr rasch, wobei sich Entwicklungen des italienischen Parteiensystems begünstigend auswirkten.

Die besonderen Gegebenheiten der nördlichen Regionen sind umfassend analysiert worden. Zahlreiche Studien haben ein Bündel von Krisenfaktoren in den Vordergrund gestellt: Die Säkularisierung, die mit einer Loslösung der Bindungen an die DC einherging; das politische Gefühl der Ausgrenzung trotz einer doch deutlichen wirtschaftlichen Zentralität der nördlichen Regionen, verbunden mit dem klassischen sozialen und psychologischen Phänomen der sogenannten „relativen Deprivation“. ${ }^{22}$ Cartocci gibt eine der umfassendsten und ver-

19 Als Ort des Schwurs von Pontida bildete sich im Jahre 1167 der Lombardenbund, der bei der Schlacht von Legnano 1176 gegen Friedrich Barbarossa kämpfte. Das Treffen auf der Pontida-Wiese wird jährlich von der LN organisiert. Die Märsche an den Po feiern den Fluss, der durch die „padanischen“ italienischen Regionen fließt.

20 Vgl. Vimercati, I lombardi, S. 10.

21 Vgl. Biorcio, La Padania promessa, S. 55.

22 Die diesbezügliche Literatur ist sehr umfangreich: Roberto Cartocci, Fra Lega e Chiesa. L'Italia in cerca di integrazione, Bologna 1994; Bull/Gilbert, The Lega Nord, und, detaillierter Ilvo Diamanti/Gianni Riccamboni, La parabola del voto bianco. Elezioni e società in Veneto (1946-1992), Vicenza 1992; Diamanti, Il male del Nord. 
ständlichsten Interpretationen dieser Elemente. Seinen Studien zufolge ist „,der Wahlerfolg der Lega Nord als eine Befriedigung der verbreiteten Integrationsnotwendigkeit zu verstehen und zwar durch die Anrufung jener lokalen Identitätsressourcen, die schon immer im zentralen und nördlichen Teil des Landes vorhanden waren". ${ }^{23}$

Es wird sich zeigen, dass der umfassende Kampf gegen die Parteien, die korrupten Mächte eines römischen Zentralismus, der wahre Grund für den Erfolg eines Protestpopulismus ist, der sich anfangs über einen Ethnonationalismus und später über einen Föderalismus mit der Identitätsdimension verbindet. Die feindliche Haltung dem Süden gegenüber (,antimeridionalismo“) wurde nicht nur von Mitgliedern und Sympathisanten der LN vertreten, sondern erreichte auch große Aufmerksamkeit durch traditionelle Parteien und Massenmedien. Was nach 1989 dann sehr typische einwanderungs- und ausländerfeindliche Züge angenommen hat, rührt bekanntermaßen aus der kontinuierlichen Polemik gegen süditalienische Arbeiter her. Die Betonung dieser Haltung hat dann maßgeblich zu einer strategischen Umstellung der politischen Achse der Lega beigetragen. Diese Umstellung wurde von Bossi als notwendig empfunden. ${ }^{24}$

Diese von der Lega eingesetzten negativen Töne dem Süden gegenüber zogen schließlich die Aufmerksamkeit der Massenmedien auf sich. Bis dahin hatten die Medien der Partei wenig Beachtung geschenkt und deshalb auch keinen Raum in den Wahlprogrammen des Fernsehens eingeräumt. So hat die Partei durch ihre neue Art populistischer Sprache eine Präsenz gewonnen, die sie sonst nicht erreicht hätte. ${ }^{25}$ Eine komplette Medienunsichtbarkeit wechselte sich mit Phasen $\mathrm{ab}$, in denen folkloristischen Elementen, der Benutzung des Dialektes in institutionellen Bereichen und der harten, fast „barbarischen“ Sprache der LN Aufmerksamkeit gewidmet wurden. ${ }^{26}$

Die mediale Darstellung der Lega hätte sich ansonsten nicht weiter entwickelt, jedoch begann die Partei im Laufe weniger Monate, bedeutende Wahlerfolge zu erringen. Insbesondere profitierte sie vom sprichwörtlichen Erdbeben „Tangentopoli“, welches bald das gesamte italienische Parteiensystem erschütterte. Unter „Tangentopoli“ versteht man bekanntlich die Entdeckung eines geheimen Parteienfinanzierungsapparats, die im Laufe weniger Jahre zum Fall zweier der größten politischen Mächte Italiens geführt hat, nämlich der Christlich-Demokratischen und der Sozialistischen Partei. Das gesamte Parteiensystem, das auf der Vorherrschaft der DC basierte, stürzte in wenigen Monaten in eine Krise. Das dadurch entstandene Vakuum wurde in den folgenden Jahren

23 Cartocci, Tra Lega e Chiesa, S. 202.

24 Vgl. Luciano Costantini, Dentro la Lega. Come nasce, come cresce, come comunica, Rom 1994, S. 47.

25 Vgl. Roberto Biorcio, The Lega Nord and the Italian Media System. In Gianpietro Mazzoleni/Julianne Stewart/Bruce Horsfield (Hg), The Media and Neo-Populism. A Contemporary Comparative Analysis, Westport 2003, S. 71-94.

26 Vgl. Tarchi, L'Italia populista, S. 142. 
teilweise von der LN und ab 1994 auch von Silvio Berlusconis neugegründeter Partei „Forza Italia“ gefüllt.

Wahlstudien, ${ }^{27}$ die seit den ersten Erfolgen der Lega Nord erstellt wurden, zeigen, dass die Lega - hauptsächlich zwischen 1989 und 1992 - die bedeutende Fähigkeit besaß, ,in den großen leeren, aus der Krise der Massenpartei entstehenden Raum“ einzudringen, „in den nördlichen Regionen sind die Stimmen der $\mathrm{LN}$ von $3,7 \%$ bis auf $17,3 \%$ gewachsen “. ${ }^{28}$

In erster Linie verlor die DC ihre Stimmen an die neuen Parteien, aber ein Teil der Stimmen der LN auch aus der Opposition. ${ }^{29}$ Abgelegt wurden die Bezüge zum Dialekt und zu den Plattformen für eine Neubewertung der kulturellen Eigenheiten der nördlichen Regionen. Die Partei konzentrierte sich nun auf die von Diamanti beschriebene „Sprache der Innovation und der Unterscheidung". ${ }^{30}$ Letztere hatte die Absicht, Kernthemen der neuen strategischen Umwandlung der Lega in den Vordergrund zu rücken, also den Protest gegen Parteien und Institutionen. Die Opposition gegen jeglichen Zentralismus, AntiPolitik, die Gegenüberstellung von Volk und Elite sowie eine einwanderungsfeindlichen Perspektive stellten bald eine erfolgversprechende Kombination für die Partei dar. ${ }^{31} \mathrm{Im}$ Jahr 1993 erreichte die Lega den vorläufigen Höhepunkt ihrer Entwicklung mit der Eroberung des Mailänder Bürgermeisterpostens durch Marco Formentini.

Die Erfolgsgeschichte der Lega kann nicht ohne die Krisensituation „Tangentopoli“ und die wachsende Verachtung gegenüber den traditionellen Parteien verstanden werden. Erst der durch diese Faktoren erreichte Erfolg ermöglichte, dass sich die Zustimmung für die LN gewissermaßen als seismischer Prozess von den ursprünglichen Erfolgsprovinzen zu den umliegenden Gebieten, d.h. von den Alpenregionen bis nach „Padanien“, ausweitete. ${ }^{32}$ Jedoch konnte man schon mit den Wahlen von 1994, als die Lega in die Berlusconi-Regierung eintrat, eine Art „Sogwirkung“ des Erfolgsphänomens erkennen.

27 Vgl. Renato Mannheimer (Hg.), La Lega Lombarda, Mailand 1991, hier vor allem die Beiträge von Paolo Natale, Lega Lombarda e insediamento territoriale: un'analisi ecologica, S. 83-12, und Mannheimer, Chi vota Lega e perché, S. 122-158.

28 Vgl. Biorcio, La Padania promessa, S. 62.

29 Vgl. ebd.

30 Diamanti, La Lega, S. 23.

31 Vgl. Piero Ignazi, Extreme Right Parties in Western Europe, Oxford 2003, S. 56.

32 Vgl. Ilvo Diamanti, La Lega Nord, dal successo alla secessione. In: ders./ Marc Lazar, Stanchi di miracoli, Mailand 1997, S. 221-235. 


\section{Regionalistischer, Identitäts- und Protestpopulismus}

An diesem Punkt möchte ich die populistischen Eigenschaften hervorheben, welche die LN in kurzer Zeit zu einem derart wichtigen Akteur der italienischen Parteienlandschaft gemacht haben. Besondere Aufmerksamkeit gilt dabei dem Verständnis einer besonderen Art von Populismus, dem die Lega zugeordnet werden kann.

\section{Die Parteienfeindlichkeit und die Antipolitik}

Wie beobachtet, waren die Parteienfeindlichkeit und generell die antipolitische Kritik zwei notwendige Elemente für den Erfolg der LN im politischen System Italiens. Die Verbreitung dieser Stimmung wurde einerseits begünstigt durch eine Krise des Parteiensystems infolge der Tangentopoli-Skandale, andererseits durch eine besondere Situation, die oben als „Nordfrage“ beschrieben wurde. Die sogenannte „Nordfrage“ hat es ermöglicht, dass sich die anti-zentralistischen und fremdenfeindlichen Botschaften unschwer in Norditalien festsetzten konnten. An diesem Punkt erscheint es angebracht, den Begriff des Volkes zu beschreiben, dem sich die LN in ihrer Anfangsphase zugewendet hat. Bekanntlich hat sich die Partei eine Art Bezugsbevölkerung für ihren Aufruf geschaffen. Im Rahmen der Interpretation des Populismus als Ethnos- oder Demos-Mobilisierung gestaltet sich die Ex novo-Erfindung des Padania-Konzeptes und des Bezugsvolkes der Padanier als Volk im Sinne von „Ethnos“. ${ }^{33}$ Die Gemeinsamkeit der materiellen und territorialen Interessen gibt der populistischen Mobilisierung durch den Bezug auf die Gemeinschaft Form und Substanz. Jene Gemeinschaft ist gleichzeitig „heartland“, ${ }^{34}$ imaginär und ersonnen, ${ }^{35}$ eine Nation für die man Unabhängigkeit fordert, eine Gesamtheit von Bezügen auf eine mythische Vergangenheit. ${ }^{36}$ Genauso gilt der Bezug aber auch einer Zukunft, die - je nach Entwicklungsphase der Lega - durch Sezession, Devolution oder Föderalismus gestaltet ist. Und obwohl dem Aufbau dieser Idee von Gemeinschaft, die wie im nüchternsten Populismus, ${ }^{37}$ nicht von Klassenunterschieden durchströmt ist, keine Idee eines padanischen Volkes ${ }^{38}$ entspricht, sind während dieser ersten Phase die Anfragen einer territorialen Ritualmobilisierung seitens der Lega, die damit ihre Aktion legitimieren wollte, selten unbefriedigt geblieben.

33 Mény/Surel, Populismo e democrazia, S. $191 \mathrm{ff}$.

34 Paul Taggart, Il populismo, Troina 2002, S. 157-163.

35 Vgl. Benedict Anderson, Imagined Communities, London 1983.

36 Vgl. Marco Aime, Verdi Tribù del nord. La Lega vista da un antropologo, Rom 2012, S. $17 \mathrm{f}$.

37 Vgl. Loris Zanatta, Sul nucleo forte di un'ideologia debole. In: Polis, 16 (2002), S. 263-294.

38 Vgl. Tarchi, L'Italia populista, S. 152. 
Das Volk, auf das sich die Lega während dieser ersten Phase bezieht, ist das Volk der ehrlichen und produktiven Arbeiter des Nordens, Opfer der Verschwendungssucht und der Verteilungsungerechtigkeiten eines römischen Zentralismus. Es handelt sich insofern um ein typisches Bezugsvolk für den Populismus, als man sich auf den gewöhnlichen Menschen mit seinem gesunden Menschenverstand beruft, der zugleich als überlegen angesehen wird. Es handelt sich um ein Volk, das auf der Suche nach einem besseren Weg ist, um die Befreiung von einem repressiven Zentrum zu erreichen; dieses Volk findet in seinem Führer einen Vertreter, der besser als andere sein Interesse verstehen und vertreten kann, indem er sich als Vermittler zwischen dem Volk und der Partei, aber keineswegs als Schöpfer darstellt.

Auf diese Art und Weise kommt eine populistische Kritik gegen die repräsentative Demokratie zustande, deren klassische Mittler, die Parteien, jegliche Fähigkeit verloren haben, mit ihrem „Volk“ in Kontakt zu kommen. Nicht durch Zufall bezeichnet die Lega Nord sich selbst als Bewegung und nicht als Partei, nicht durch Zufall verlässt sie sich auf Umberto Bossi sowohl im Blick auf die Entwicklung der politischen Strategie als auch der Organisation und Verwaltung der eigenen Strukturen. Es handelt sich bei ihm um einen politischen Führer (Leader), der den Weg zeigt, Entscheidungen trifft, sich von seinen Mitarbeitern und Ideologen ${ }^{39}$ befreit, das letzte Wort in der Frage hat, wer legitimerweise als Teil des padanischen Volkes betrachtet werden kann und insbesondere, wer das Recht hat, in der Bewegung aktiv mitzuwirken.

Im Rahmen der Feindidentifizierung entsteht dann die Grenze zwischen Volk und Nicht-Volk. Währenddessen entsteht immer genauer auch der antipolitische Verweis. Während dieser ersten Lebensphase der LN sind die Feinde die Parteien der Ersten Republik sowie die institutionellen Akteure, die den römischen Zentralismus vor den Angriffen der Bewegung schützen. Es handelt sich um diejenigen, die „da oben“ agieren und keine Rücksicht auf die Bedürfnisse dieses wahren Volkes nehmen. Das wahre Volk ist dasjenige, welches Reichtum und Wohlstand erzeugt, im Gegensatz zum faulen „Dieb“ aus dem Süden.

\section{Die Anti-Einwanderungshaltung als Mittel der Identitätskonstruktion}

Während dieser Phase beginnt der Ausschluss derjenigen, die nicht zur Gemeinschaft gehören. Es handelt sich um den unproduktiven Süditaliener, der keinerlei Beitrag zum Wohlstand des Gemeinwesens geleistet hat und daher nicht in dieses einbezogen werden darf. Ähnlich wie später bezüglich der Haltung zur außereuropäischen Einwanderung, identifiziert die Lega die Feinde des Volkes durch eine ausschließende Logik: Da es nicht möglich ist, die Grenzen einer

39 So geschehen mit Gianfranco Miglio, dem Anreger des föderalistischen Projektes der Lega, der 1994 aus der Partei gedrängt wurde. 
padanischen Identität genau zu ziehen (einschließende Logik), wird eine gegenteilige Strategie verfolgt. Die Bestimmung des Nicht-Volks entsteht durch die Identifikation derjenigen, die freiwillig oder unfreiwillig, aus dem natürlichen Zugehörigkeitsrahmen, der als einziger der Gemeinschaft den Zugehörigkeitssinn gibt, ausgeschlossen werden können.

\section{Die traditionellen Werte}

Auf diesem Wege des Ausschlusses nehmen auch die Bezüge auf die gemeinschaftlichen Werte Form und Substanz an. Im Rahmen eines mythischen Entstehungsprozesses in der Vergangenheit kommen die traditionellen Bezüge zustande und es werden Rituale erfunden, wie etwa die Treffen in Pontida, die Märsche an den Fluss Po und die Benutzung einer keltischen-traditionellen Symbolik. Während des Umwandlungsmoments von der Ersten zur Zweiten Republik, deren Hauptmerkmale Deideologisierung und Säkularisierung sind, ermöglichen diese Rituale der Lega die Propagierung eines Traums von einem versprochenen Land, das eine Alternative bietet zu „einer kalten, erzählungsarmen Politik, die nichts weiter als administrative Routine ohne besondere ideologische Trennungen ist und immer weniger in der Lage erscheint, Ideale oder neue beziehungsweise alternative Verwaltungsmodelle vorzuschlagen." ${ }^{40}$ Traditionelle Werte, welche die LN während dieser Periode propagiert, sind also die Werte einer fleißigen Gemeinschaft, die sich für die Mehrung des Wohlstands der nördlichen Bevölkerung und eine damit verbundene Abweisung des bestehenden Individualismus der Parteien einsetzt, denn diese Parteien bewirken Separierung, anstatt eine Union zur Förderung des Gemeinwohls herbeizuführen.

Es handelt sich um ein Gemeinwohl, dessen Empfänger die Angehörigen einer territorialen Bezugsgemeinschaft sind. In diesem Sinn kann man die Perspektive der Änderung der territorialen Ordnung verstehen: Die Lega ist der erste Akteur, der aus „dem in der Geschichte der Republik vermiedenen Bruch zwischen Zentrum und Peripherie das Hauptthema seines Strebens und seiner Identität macht. "41 Wie bekannt, stellt während der gesamten 1980er Jahre der Föderalismus das Fundament dar, auf dessen Grundlage die Lega das Thema der territorialen Ordnung diskutiert. Dies ermöglicht der Lega „das Vertrauen gewisser gemäßigter Gesellschaftsschichten und einiger Sektoren der städtischen Mittelschicht zu gewinnen. “42 Die bürgerliche Gesellschaft imaginiert die Lega als ein Mittel der Innovation, welches in der Lage ist, Druck auf einen Staat und ein politisches System auszuüben, das keinerlei Willen zur Veränderung

40 Aime, Verdi Tribù, S. 31.

41 Diamanti, Mappe dell'Italia politica, S. 70.

42 Diamanti, Il male del Nord, S. 64. 
mehr besitzt. Durch das Konzept des Föderalismus gelingt es der Lega, sich vom hauptsächlich kulturellen Lokalismus der ersten Phase zu lösen.

Während der Phase zwischen ihrer Gründung und dem Anfang der 1990er Jahre steht die Lega also für einen mannigfaltigen Populismus. Es handelt sich einerseits um einen regionalistischen Populismus ${ }^{43}$ im Blick auf die territoriale Dimension und die lokalistischen Bestrebungen, anderseits um einen Identitätspopulismus, der sich auf das Bild eines produktiven Nordens im Kampf gegen das räuberische Zentrum und den faulen Süden stützt. Es handelt sich aber auch ganz wesentlich um einen Protestpopulismus, der seine Existenzgrundlage aus der manichäischen Gegenüberstellung zwischen Volk und wirtschaftlichen bzw. politischen Eliten der Ersten Republik bezieht. All dies geschieht im Rahmen einer Phase, in der die Partei die Entscheidung zur Teilnahme an der ersten Berlusconi-Regierung trifft. Bis zu diesem Moment hatten populistische Charakteristika die Aufmerksamkeit der öffentlichen Meinung und der Medien auf sich gezogen. Im folgenden Abschnitt wird gezeigt, wie der abnehmende Rückhalt in der Bevölkerung, den die Konkurrenzsituation mit der neugegründeten Partei Berlusconis nach sich zog, zu einer Radikalisierung der einwanderungsfeindlichen Perspektive und der territorialen Ordnungsorientierung führte. Dies wurde als der einzige Ausweg aus der Sackgasse angesehen, in der sich die Partei nach ihrer ersten Regierungsbeteiligung befand.

\section{Wandlung des Populismus im Radikalisierungsprozess und in Konkurrenz zu Silvio Berlusconi}

Mit der Gründung von Berlusconis Forza Italia (FI) und mit dessen Entscheidung, in die politische Arena einzutreten, begann für die LN eine sehr schwierige Phase. Der neue politische Akteur wollte die Leere füllen, die aus der Krise der zwei größten Parteien der Ersten Republik entstanden war. FI positionierte sich im Mitte-Rechts-Umfeld und rechtfertigte die eigene Existenz mit der Behauptung, eine wirklich neue, liberale Partei zu repräsentieren. Berlusconis Insistieren auf der Notwendigkeit, die Modalitäten der Parteiherrschaft der Ersten Republik zu überwinden, die Kritik an den Berufspolitikern, das Evozieren seiner eigenen unternehmerischen Erfolge als Erfolgsgarantie und die gleiche territoriale Verortung des Antriebs des neuen politischen Projektes in Mailand und der Lombardei bewirkte eine thematische Überlagerung mit den Themen der LN.

Am Vorabend des Falles der Ersten Republik war die LN nun, geschädigt durch das Inkrafttreten eines neuen Wahlgesetzes, das schon seit den Wahlen

43 Vgl. Roberto Biorcio, Il populismo regionalista della Lega Nord. In: Democrazia e Diritto, 2 (1992), S. 239-256. 
von 1994 offensichtliche Auswirkungen hatte, nicht mehr nur die einzige Vertreterin eines antipolitischen und parteienfeindlichen Diskurses. In Form der FI erwuchs ihr auch eine neuartige Konkurrenz durch eine andere Art des Populismus. Einige Kommentatoren bezeichneten diese neue Form als einen „Telepopulismus" ${ }^{44}$ andere erblicken darin eher eine plebiszitäre Haltung, die im ständigen Abgleich mit dem sogenannten „Willen des Volkes“ ihr Fundament hat. ${ }^{45}$ Hauptsächlich musste sich die LN jedoch zunehmend auch Berlusconis Konkurrenz bezüglich der Themen Steuern, Bürokratie und der Unterstützung mittelständischer Unternehmen stellen. ${ }^{46}$

Hatten die Wahlen von 1992 den Erfolg der antipolitischen und regionalistischen Strategie der LN begründet, beinhalteten jene des Jahres 1994 nach dem Aufritt Berlusconis für Bossis Partei das Risiko der Isolation. Denn die neuen Wahlgesetze zwangen den Parteien die Logik von Wahlallianzen auf, welche die LN mit dem Kompromiss einer wechselhaften Koalition mit FI akzeptieren musste. ${ }^{47}$ Der Eintritt in die aus FI, CCD (Centro Cristiano Democratico) und AN (Alleanza Nazionale) zusammengesetzte Regierungskoalition war für die Lega ein schwieriger Schritt. So fand sich die Partei in einer zwanghaften Koalition mit einer politischen Formation wie der AN wieder, welche sich der Idee des Nationalstaats verpflichtet fühlte. Von vergleichbarer Komplexität waren die Beziehungen mit Berlusconi, da sich zunehmend abzeichnete, dass FI gerade in jenen Gebieten, in denen die Lega 1993 triumphiert hatte, ein starker Konkurrent war. Zudem wurde die Möglichkeit immer geringer, föderalistische Forderungen umzusetzen, was offiziell die Regierungsteilnahme der Lega rechtfertigte. Diese Phase der Lega als Partei sowohl des Kampfes als auch der Regierung wurde abrupt mit Bossis Entscheidung beendet, die Koalition mit FI nach nur acht Monaten abzubrechen. Bossis Strategie, in die Opposition zurückzukehren, führte zu starken parteiinternen Konflikten und hatte zur Folge, dass wichtige Vertreter, auch solche ersten Ranges, die Lega verließen oder ausgestoßen wurden.

Bossis Strategie sah vor, durch eine Rückkehr in die Opposition den Weg für die föderalistische Perspektive zu ebnen und das Projekt Padanien zu erweitern. Erreicht werden sollte dies einerseits über die Schaffung von Traditionen und andererseits über die Konstruktion entsprechender Symbole und Institutionen. Es ist dies die Phase, in der das sezessionistische Projekt entsteht, in der Riten und Mythen der Erfindung Padaniens vermittelt werden. Dabei wurde auf Kommunikationsmedien gesetzt wie die 1996 gegründete Parteizeitung La Padania,

44 Pierre-André Taguieff, L'illusion populiste, Paris 2002.

45 Vgl. Tarchi, L'Italia populista, S. 172.

46 Vgl. Biorcio, La rivincita del Nord, S. 18.

47 Im Norden bildete FI eine Koalition mit der Lega und dem CCD (Polo delle Libertà), im Süden mit AN, der von Gianfranco Fini geführten Partei, und dem CCD (Polo del Buon governo). 
den Radiosender Radio Padania Libera ${ }^{48}$ und den Fernsehkanal Telepadania, der 1998 auf Sendung ging. Das Netzwerk von Nebenorganisationen sollte nun nicht länger föderalistische Ideale, sondern das Ideal der Unabhängigkeit Padaniens verbreiten.

Durch den Austritt aus der Koalition versuchte die Lega also zunächst, sich von der FI zu unterscheiden. Weiterhin bestand die Absicht, die Organisation der Bewegung um Bossi zu gruppieren und die Wählerbasis neuerlich zu mobilisieren.

Die Strategie, alleine anzutreten, brachte Bossis Partei 1996 einen Wahlerfolg $(10 \%)$ ein und verhinderte den Sieg der Mitte-Rechts-Koalition. Von diesem Zeitpunkt an und bis zur Entscheidung von 2001, sich wiederum mit Berlusconi zu verbünden, begann die Lega ihren ursprünglichen Protestpopulismus radikal zu interpretieren. Damit positionierte sie sich klar gegen „Roma Polo“ und „Roma Ulivo“.49 1995 wurde das Parlament des Nordens gegründet; im Jahr darauf die sogenannten „Camicie Verdi“ (Grüne Hemden), die bisweilen als eine Art von Sicherheitsdienst präsentiert wurden, manchmal aber auch als paramilitärische Gruppe, die im Fall eines Sezessionskampfes aktiv werden sollte. 1996 schlug Bossi die Schaffung eines Komitees zur Befreiung Padaniens vor. Dessen Gründung wurde während des alljährlichen Treffens der Parteimitglieder in Pontida zusammen mit einer Regierung derselben Region angekündigt. Parallel zu populistischen Parteien des Alpengebietes, ${ }^{50}$ die mit diesen Themen wenig später einen nahezu explosionsartigen Wahlerfolg erlebten, fing seinerzeit auch die LN an, sich gegen die Einwanderung und die staatliche Steuerpolitik zu positionieren sowie für „law and order" einzutreten. Folgende Faktoren haben in dieser Phase zu einer Betonung von Merkmalen geführt, wie sie auch für jene populistischen Parteien typisch sind, die im zeitgenössischen europäischen Szenario ihre Ursprünge im Umfeld der radikalen Rechten hatten: Das von der Parteispitze zunehmend instrumentalisierte Thema der Ausländerfeindlichkeit, welches auf diese Weise gewissermaßen zum Eigentum der Partei wurde; außerdem sezessionistische Szenarien, welche die Partei durch das Handeln und die Rhetorik der Leadership zusammen mit einem komplexen Apparat von Nebenorganisationen in Szene setzte. Dieser Kurs wurde in erster Linie von parteinahen Medien begleitet und unterstützt. Betrachten wir nun also kurz die wichtigsten

48 Zur Rolle von Radio Padania Libera siehe: Gianluca Croce, Il mulino della Lega. Come Radio Padania Libera forma l'opinione del popolo leghista. In: Comunicazione Politica, 1 (2007), S. 129-140.

49 Polo steht für Polo delle Libertà, die Mitte-Rechts Wahlkoalition, welche 1994 von Berlusconi gegründet wurde. Ulivo (Olivenbaum) ist der Name der Mitte-Links-WahlKoalition, 1995 aus mehreren Mitte-Links-Parteien gegründet. Romano Prodi war eine ihrer Leitfiguren. Die Ausdrücke „Roma Polo“ und „Roma Ulivoj“ sind ein klassisches Beispiel der Rhetorik der LN. Beide Koalitionen (Mitte-Rechts und Mitte-Links) werden durch den Bezug auf Rom herabgewürdigt.

50 Vgl. Bruno Luverà, Il neopopulismo alpino, Mailand 2000. 
Eigenschaften des Populismus jener Periode der LN mit Blick auf die soeben angesprochenen Aspekte.

Parteienfeindlichkeit und Anti-Politik bezeichnen in dieser Phase der Geschichte der Lega eine stärkere Akzentuierung der Opposition zum Establishment. „Roma Polo“ und „Roma Ulivo“ verkörpern dabei die alte Parteienherrschaft und Berlusconis „nuovismo“, d. h., die Strategie der Neuheit in Absetzung vom Etablierten, eine Mystifizierung der wahren Ursachen des Koalitionsbruchs. Die Ablehnung oder Verachtung zentralistischer Institutionen und die antiinstitutionelle Haltung erreichten in dieser Periode ihren Höhepunkt sowohl im Rahmen der alltäglichen Parteiaktivität als auch hinsichtlich der vielen zusätzlichen Initiativen, mit denen die Idee eines eigenständigen Padaniens verbreitet wurde. So hatte etwa der von der Partei vorgebrachte Vorschlag, „Schatten“-Institutionen (wie das padanische Parlament und die padanische Regierung) zu bilden, das Ziel, die zentralen Institutionen zu delegitimieren. Darüber hinaus wurden die Magistrate hart dafür gescholten, dass sie die aus Sicht der Lega legitimen Unabhängigkeitswünsche des Nordens konterkarierten. Um ihr Unabhängigkeitsstreben und das Projekt einer Reform bezüglich der territorialen Ordnung zu stärken, organisierte die Lega eine Volksabstimmung über die Sezession, welche einer Kritik an der repräsentativen Demokratie und der Aufwertung direkter Demokratie dienen sollte. Die Unterschriftensammlung erfolgte unter Pavillons (gazebo), die auf Straßen und auf Plätzen eingerichtet wurden.

Die Expansion der Lega während dieser Phase basiert jedoch nicht ausschließlich auf der beschriebenen Positionierung der Partei gegenüber dem Establishment. Zur steigenden Popularität der Bewegung trugen ihre Rolle als Oppositionspartei, die es ihr ermöglichte sich als die einzige Stimme des padanischen Volkes darzustellen, sowie ihre einwanderungsfeindliche Haltung bei. Wie gezeigt, hatte die Rhetorik gegen die „terroni“ (die faulen Süditaliener) in der ersten Phase den Vorteil, dass eine Beziehung zwischen dem faulen Süden und dem fehlenden Willen der süditalienischen Migranten im Norden, die produktive Ethik der gastgebenden norditalienischen Bevölkerung zu teilen, hergestellt werden konnte. Dabei wurden außerdem Mafia und politisches Establishment ${ }^{51}$ gleichgesetzt - mit dem Ziel, gleichzeitig den Süden und die herrschenden Parteien anzugreifen. In der neuen Phase sind es dagegen die nicht-europäischen Ausländer, die ins Visier des identitätsstiftenden Populismus der Lega geraten. Die gegen Immigranten gerichtete Polemik wurde dadurch erleichtert, dass Einwanderungswellen aus Nicht-EU-Staaten in Italien seit Anfang der 1990er Jahre stetig zunahmen und dass dies gerade der nördlichen Bevölkerung Italiens, wie anhand von Meinungsumfragen gezeigt werden konnte, Sorgen bereitete. ${ }^{52}$

So organisierte die Lega Demonstrationen gegen die Nomadensiedlungen der "Zigeuner", Roma und Sinti, plante auf lokaler und nationaler Ebene Initiativen gegen die „wilde“ Einwanderung und organisierte 1999 eine Unterschriften-

51 Vgl. Tarchi, L'Italia populista, S. 148.

52 Vgl. Biorcio, La rivincita del nord, S. 57. 
sammlung gegen das Einwanderungsgesetz (das sogenannte Turco-NapolitanoGesetz), das von der vorhergehenden Mitte-Links-Regierung erlassen worden war. Die Anti-Einwanderungs-Rhetorik wurde durch die klassischen Perspektiven des Wohlstandschauvinismus und durch die symbolische Beschwörung einer homogenen Kollektivität gerechtfertigt. In dieses Kollektiv findet der außereuropäische Ausländer nur dann Aufnahme, wenn er sich integriert und produktiv ist, wohingegen kein Platz vorhanden ist für kulturelle oder wertebedingte Abweichungen, die nicht den Eigenheiten des padanischen Volkes entsprechen. Im Bereich der traditionellen Werte steht deshalb auch der Schutz jener Sphäre im Vordergrund, welche die größten Identifikationspotentiale birgt: der Familie. Nicht zufällig findet sich im Wahlprogramm des Jahres 2001 die Behauptung: „Die Krise der Familie und das folgende Verschwinden einer sozialen Bindung hat unsere Gesellschaft geschwächt. Es handelt sich um die Gesellschaft, die durch verwüstende Einwanderungswellen komplett verändert und zerstört werden könnte. " 53 Noch stärker ausgeprägt ist nun die Kritik am Individualismus, die wie oben gezeigt, schon in der ersten Entwicklungsphase der Partei propagiert wurde. So heißt es im selben Wahlprogramm von 2001, dass „der Individualismus für eine nur innerliche und gefühlsmäßige Auffassung der Familie verantwortlich ist und dadurch die Familie von der Gesellschaft trennt. [...] Die individualistische Gesellschaft hat sich auf die individuellen Rechte und nicht auf die Familienrechte konzentriert, woraus eine soziale und zivile Irrelevanz der Familie folgt. Heute entscheidet praktisch der freie Wille jedes Mitglieds über die Verantwortung für die Stabilität des Familienkerns“. 54

Der Populismus der Lega hat sich also während dieser zweiten Expansionsphase gewandelt. Ohne Zweifel handelt es sich nun in erster Linie um eine Form von Identitätspopulismus, in dem die Grenzen des heartland eher heraufbeschworen denn identifiziert werden. Die Idee des padanischen Volkes, auf welche die Lega Bezug nimmt, wird dabei über zwei Modi aufgebaut: Einerseits über die Evokation einer mythischen Vergangenheit und andererseits durch die systematische „Zerstörung“ der historischen Ursprünge eines unitarischen italienischen Staates. Vermittelt werden diese Gedanken hauptsächlich über die Parteizeitung „La Padania“ wie auch durch weitere Publikationen, welche sinnbildliche Namen tragen wie „Quaderni Padani“ und „Sole delle Alpi“. Obwohl die Partei offiziell nicht beabsichtigte, sich den Positionen der rechten populistischen Parteien Europas anzunähern oder anzupassen, teilte sie mit diesen fortan den Großteil ihrer Programmpunkte: Eine an Xenophobie grenzende einwanderungsfeindliche Haltung; das Insistieren auf Sicherheit und den Kampf gegen Kriminalität; erste Anzeichen der Idee einer Notwendigkeit des Schutzes der kulturellen Wurzeln Europas gegen die außereuropäische Einwanderung. Weiterhin beinhaltet diese Feindschaft einen Skeptizismus bezüglich der Globalisierung wie auch gegenüber einer Europäischen Union, die nicht auf dem Ziel

53 Parteiprogramm der Lega Nord, 2001.

54 Parteiprogramm der Lega Nord, 2001. 
eines Europa der Völker fußt, sondern hauptsächlich wirtschaftliche und bürokratische Aspekte in den Mittelpunkt stellt. ${ }^{55}$ Sie beinhaltet ebenso einen ökonomischen Liberalismus, begleitet von einer stetigen Kritik an der Macht des Zentralstaates. All diese Elemente machen aus der Lega einen typischen Vertreter eines rechten Populismus, wie er zu jener Zeit in verschiedenen europäischen Ländern große Wahlerfolge erzielte. ${ }^{56}$ Wie im Falle des Front national in Frankreich oder der Freiheitlichen Partei Österreichs, so entsteht auch im Falle der Lega eine Debatte über die rechtsradikale Natur der Partei. Zu den oben genannten Elementen hinzu kommt noch die innere Organisation der Partei, die stark von ihrer Führerpersönlichkeit beeinflusst wird. Die LN steht und fällt mit ihrem Kopf Bossi: Er legt die Strategie fest, entscheidet wann Allianzen geschlossen und gebrochen werden und bestimmt, ob ausgeschlossen wird, wer sich den Vorschriften des Sekretärs nicht fügt. ${ }^{57}$ Diese Elemente haben dazu geführt, dass hauptsächlich ausländische Beobachter, die bisweilen dazu tendieren die Ursprünge einer Zentrum-Peripherie-Kluft zu unterschätzen, die Lega am extremen Ende eines klassischen Rechts-Links-Kontinuums verorten. Es handele sich um eine modernisierte Version des Rechtextremismus, Ignazi spricht daher auch von einem „post-industriellen Rechtsextremismus“. ${ }^{58}$

\section{Von der Institutionalisierung bis zur Expansion im Jahr 2008}

Der große Wahlerfolg der LN von 1996, der den Anfang der zweiten Expansionsphase der Partei markiert, war nur von kurzer Dauer. Bereits die Europawahlen von 1999, bei denen die LN nur 4,5\% erzielte, stellen den Beginn einer Krise dar. Verschiedene Meinungsumfragen verdeutlichten seinerzeit, dass die Idee der Padania unter der nördlichen Bevölkerung weder verbreitet noch begriffen worden war. ${ }^{59}$ Zudem wurde das Projekt selbst unter denen, die dessen potentielle Vorteile erkannt hatten, nur von einer Minderheit unterstützt. In der Tat waren es andere Themen - etwa Arbeitslosigkeit, Steuern, Einwanderung und Kriminalität -, welche potentielle Wähler der Partei Ende der 1990er Jahre beschäftigten. ${ }^{60}$

55 Über die Entwicklung der Haltungen der LN bezüglich europäischer Themen und allgemein über ihre Außenpolitik siehe Marco Tarchi, The conflicting foreign agenda of the Alleanza Nazionale and the Lega Nord. In: Christina Schori Lang (Hg.), Europe for the Europeans. The Foreign and Security Policy of the Populist Radical Right, Burlington 2007, S. 187-208.

56 Vgl. Betz, Radical Right-wing Populism.

57 Vgl. Artikel 53 „Statuto della Lega Nord per l'indipendenza della Padania“, 2002.

58 Piero Ignazi, L'estrema destra in Europa, Bologna 1994, S. 51-53.

59 Vgl. Bull/Gilbert, The Lega Nord and the Northern Question, S. 116 f.

60 Vgl. Biorcio, La rivincita del Nord, S. 42 f. 
Nach dem Föderalismus und der Sezession kehrte die Partei infolgedessen wieder zum Konzept der Devolution ${ }^{61}$ zurück. Die Mission eines sezessionistischen Kampfes zeigte das immer deutlichere Interesse der Partei für typische Themen europäischer populistischer Parteien - Themen, welche die Wähler am meisten zu beschäftigen schienen und bei denen sich die Lega innerhalb Italiens ohne nennenswerte Konkurrenz wusste; dies gilt insbesondere für das Thema der außereuropäischen Einwanderung. ${ }^{62}$

Nicht länger haltbar und der Basis schwer zu vermitteln war auch die Position der Isolation, in welche die Lega 1996 geraten war, nachdem sie entschieden hatte, bei den Wahlen alleine anzutreten. Dies galt ebenso für eine Wählerschaft, die sich 1999 deutlich von der Partei entfernt hatte. Ab dem Jahre 2000 wurden folglich die Verhandlungen mit FI wieder aufgenommen. Sie endeten in der Wahlallianz für die Regionalwahlen desselben Jahres und für die Parlamentswahlen von 2001, bei denen die Lega einen Stimmenzuwachs verzeichnete. Obwohl die Lega bei den Parlamentswahlen 2001 noch nicht einmal 4 \% (3,9\%) erreicht hatte, blieb sie während der gesamten Legislaturperiode der Allianz mit Berlusconi treu, was sie damit begründete, dass dieser Weg die einzige Möglichkeit sei, die Ziele der Bewegung zu verwirklichen - vom Föderalismus bis zur Verschärfung der Einwanderungsgesetzgebung. In der Tat wurde schon im Jahr 2002 ein neues Einwanderungsgesetz erlassen, das den Namen „Bossi-Fini“ erhielt. Benannt wurde es nach dem Kopf der Lega, seinerzeit Minister für institutionelle Reformen, und dem Sekretär der Alleanza Nazionale und Vizepräsident des Ministerrates, Gianfranco Fini. Dieses Gesetz verschärfte unter vielen Aspekten das bis dahin geltende Turco-Napolitano-Gesetz. Seine Durchsetzung wurde von der Lega als Beleg für ihre erfolgreiche Regierungsbeteiligung hingestellt. Abermals verfolgte die Partei so das Ziel, sich ihren Wählern als „Lega di Lotta e di Governo“ zu präsentieren. ${ }^{63}$ Das eigentliche Ziel der Lega, der Föderalismus, wurde während der Legislaturperiode von 2001 bis 2006 allerdings de facto nicht erreicht. Eine erste Reform, die sich dem Thema Förderalismus widmete, war bereits in der vorherigen Legislaturperiode von der Mitte-LinksRegierung verabschiedet und durch eine Volksabstimmung im Jahre 2001 bestätigt worden, bei welcher die Lega ihrer Anhängerschaft jedoch dazu aufgefordert hatte, mit „Nein“ abzustimmen. Dies geschah mit der Begründung, bei dem in der Gesetzesnovelle vorgeschlagenen Föderalismus handele es sich um einen

61 Die LN bezieht sich mit „Devolution“ auf die Übertragung administrativer Funktionen vom Souverän an die Gebietskörperschaften auf das Vorbild Großbritanniens.

62 Wie von Tarchi beobachtet: ders., L'Italia populista, S. 150. Obwohl sich die AN im rechten Spektrum des Rechts-Links-Kontinuums positionierte, konnte sie nicht zu sehr auf dem Thema Einwanderung aufbauen, aus Angst, die Xenophobie-Vorwürfe würden die alte Polemik über die faschistische Natur der Partei Finis wiederbeleben.

63 Vgl. Daniele Albertazzi/Duncan McDonnell, The Lega Nord in the second Berlusconi Government: in a League of its own. In: West European Politics, 28 (2005), S. 952-972. 
„Vorgetäuschten“. ${ }^{64}$ Während der Legislaturperiode von 2001 bis 2006 wurde das Thema der Reform im Sinne eines föderalistischen Staates von der MitteRechts-Regierung erneut angegangen. Dem Parlament gelang es zunächst, den Reformvorschlag mit einer einfachen Mehrheit zu verabschieden, jedoch wurde dieser bei dem zur Bestätigung der Gesetzesnovelle angesetzten Referendum im Jahr 2006 verhindert. So gelang es der Lega nicht, ihr zentrales Ziel zu erreichen. ${ }^{65}$

Während der folgenden kurzen Legislaturperiode, in der von 2006 bis 2008 eine Mitte-Links-Koalition an der Spitze des Staates stand, blieb die Lega in der Opposition, wo sie sich auf eine neuerliche Allianz, dieses Mal mit Popolo delle Libertà (PDL) vorbereitete. Diese neue politische Formation, die aus FI und AN zusammengesetzt war, entstand 2009. Just zu den Wahlen des Jahres 2008 erlebte die Lega eine neue Phase der Expansion. Diese lässt sich zu großen Teilen auf die Desorientierung der Wähler gegenüber dem neuen politischen Akteur (PDL) zurückführen, dem es nicht gelungen war, den Stimmenanteil der zwei Gründungsparteien zu übertreffen. Zudem dürfte das gute Abschneiden der LN auf die Arbeit der Partei in den zwei Oppositionsjahren zurückzuführen sein, während derer sie eine Aktualisierung ihrer populistischen Stammthemen vorgenommen hatte - nun qua Konzentration auf die klassische Gegenüberstellung von Volk und Elite. In der Tat erlangte die Polemik gegen die sogenannte „Kaste“ der Berufspolitiker und die von diesen verursachten Kosten im Laufe der letzten Jahre große mediale Aufmerksamkeit und wurde als Thema in der Folgezeit nicht allein von der Lega, sondern auch von anderen, nicht rechts orientierten politischen Akteuren besetzt. ${ }^{66}$

Während der Regierungsjahre versuchte die Lega, auch ihren anderen zentralen Zielen treu zu bleiben: Dem bereits oben angesprochenen Föderalismus sowie einer Politik des law and order. Bezüglich des ersten Aspektes wurde im Laufe des Jahres 2009 eine Reihe von Gesetzen zum fiskalischen Föderalismus erlassen, für welche die Partei sich sehr eingesetzt hatte. Von der öffentlichen Meinung wurden diese „Erfolge“ der Lega allerdings mit einer gewissen Gleichgültigkeit aufgenommen. ${ }^{67}$ Dem ebenfalls in dieser Zeit erlassenen Sicherheitsgesetz, dass die Einführung des Strafbestandes der illegalen Einwanderung enthielt, wurde dagegen große Aufmerksamkeit zuteil. ${ }^{68}$ Gleiches konstatieren lässt sich für die Umfunktionierung der sogenannten Centri di Permanenza Tempora-

64 Giorgia Bulli/Filippo Tronconi, The Lega Nord. In: Anwen Elias/Filippo Tronconi From Protest to Power: Autonomist Parties and the Challenges of Political Representation, Wien 2011, S. 51-74.

65 Die Anfragen des Referendums wurden nur in den Regionen Venetien und Lombardei verabschiedet, während in allen anderen Regionen das „Nein“ dominierte.

66 Der Bezug betrifft die Partei Italia dei Valori des ehemaligen Richters von Mani Pulite, Antonio di Pietro, und das Movimento Cinque Stelle des Komikers Beppe Grillo.

67 Vgl. Biorcio, La rivincita del Nord, S. 75 f.

68 Der Strafverschärfungsgrund unerlaubter Einwanderung ist dann vom Verfassungsgericht für illegal erklärt worden (Urteil 8. 7.2010 $n^{\circ} 249$ ). 
nea $(\mathrm{CPT}=$ Zentren zum Temporären Aufenthalt), die nun Centri di identificazione e di espulsione ( $\mathrm{CIE}=$ Zentren zur Identifikation und Ausweisung) genannt wurden.

Generell konzentrierte sich die Aufwertung des Regierungshandelns der LN auf die Figur Roberto Maronis ${ }^{69}$ und dessen Aktivität als Innenminister und „institutionelles Gesicht“ der LN. Die Folgen einer schweren Erkrankung Bossis im Jahr 2004 hinderten den Sekretär daran, seine Führungsrolle auszuüben und schwächten seine Position. Interne Spaltungen entstanden, die bald Parteiströmungen ähnelten und die fortan neben die seit Parteigründung bestehenden Fraktionen Venetiens und der Lombardei traten.

\section{Krise der Partei}

Die jüngste Krise der Partei entstand aus einer Vielzahl von Faktoren. Teilweise war sie vorhersehbar, teilweise kam sie unerwartet und überraschte auch den Großteil der Wähler zutiefst. Einerseits hatte die Partei sehr unter der Verbindung mit dem unbequem gewordenen Regierungspartner gelitten. So hatten die Skandale, in die Berlusconi während der letzten Monate vor seinem Amtsabschied verwickelt war, die Glaubwürdigkeit der gesamten Regierung in Mitleidenschaft gezogen. Dies gilt insbesondere für die Bewältigung der internationalen Wirtschaft- und Finanzkrise. In dieser auch äußerlich schwierigen Konstellation hat die Unsicherheit, die auch auf eine intern fragwürdig gewordene Führungsrolle zurückging, der Lega sicherlich keinen guten Dienst erwiesen. Sie zog immer wieder ernsthaft in Betracht, aus der Regierung auszutreten und in die Opposition zu gehen. Allerdings haben die Finanzskandale der letzten Monate offenbart, dass in der Partei Diebstahl, Veruntreuungen und gesetzeswidrige Parteienfinanzierung über Jahre hinweg an der Tagesordnung waren. Somit besteht gewissermaßen eine Traditionslinie zur Parteienherrschaft der Ersten Republik, aus deren Ablehnung heraus die Lega ursprünglich ins Leben gerufen worden war und auf der sie sowohl ihre Wahlerfolge als auch ihre politische Legitimität aufbaute.

Die dramatische und schnelle Entwicklung der Ereignisse, die in wenigen Monaten zum Fall der Regierung führten, haben zusammen mit den genannten

69 Roberto Maroni ist seit der Gründung eine der Schlüsselfiguren der LN. Er war der Innenminister der ersten Regierung Berlusconi und Arbeitsminister in der Legislaturperiode von 2001 bis 2006. Als nachmaliger Innenminister in der Legislaturperiode von 2008 bis 2011 repräsentierte Maroni die institutionelle Seele der Partei. Im Verlauf der letzten Legislaturperiode hat er sich auf dem Gebiet der Sicherheitspolitik und gegen die illegale Einwanderung engagiert und gelangte zu großer Popularität auch bei Nicht-LN-Wählern. Im Verlauf des letzten Kongresses der LN (Juli 2012) wurde er zum Parteisekretär gewählt. 
Faktoren verhindert, dass die Partei ihre parlamentarische Opposition gegen die Regierung Monti ausnutzen konnte. Dazu kamen medial diskutierte Skandale mit zahlreichen Details über einen Kreis von Akteuren im engsten familiären und politischen Umkreis Bossis - nicht zufällig „magischer Kreis“ genannt. Außerdem war die Partei nach zehn Jahre gezwungen, einen Föderalkongress einzuberufen, ${ }^{70}$ der Ende Juni 2012 stattfand und bei dem - freilich nicht ohne offenkundige Spannungen zwischen den nunmehr unverhüllt agierenden Parteiströmungen - der ursprüngliche Kopf der Partei, Umberto Bossi, die Führung an Roberto Maroni übergab. In seiner Antrittsrede versuchte der neue Sekretär, seiner Partei eine Strategie zur Überwindung der Krise zu diktieren. Diese basierte hauptsächlich auf einer Rückkehr zu ursprünglichen Schlagwörtern; nicht zufällig hatte man als Kongressslogan „prima il Nord“ („der Norden zuerst“) gewählt. Eine strukturelle Erneuerung der Parteiorganisation sollte dazu dienen, dieser die Fähigkeit zur Kontrolle wiederzugeben. Dies beinhaltete regionale Sekretariate (die in der Sprache der Lega als „nationale“ bezeichnet werden) und den Ansporn zur Wiederaufnahme territorialer Aktivitäten auf Basis der Sektionen. Es handelte sich um eine Aufgabe, die alles andere als einfach war, scheint es doch, als sei die Lega in diesem Moment nicht in der Lage gewesen, ihre populistischen Elemente auf eine für sie förderliche Art und Weise auszunutzen. Hatten in der Vergangenheit doch gerade jene Elemente, trotz der hier nachgezeichneten Popularitätseinbrüche, aus der Lega einen der wichtigsten Akteure des italienischen politischen Systems der letzten zwanzig Jahre gemacht.

\section{Schlussbetrachtung}

Wir haben uns im Rahmen der Beschreibung der wichtigsten Entwicklungsphasen der LN vom Ende der 1980er Jahre bis zur heutigen Krise mehrmals auf das Konzept des Populismus bezogen. An die Polysemie des Konzeptes wird immer dann erinnert, wenn man versucht, „den starken Kern einer schwachen Ideologie"71 zu identifizieren oder zu einer Art von Minimaldefinition zu gelangen. Solch eine Definition sollte einerseits für die Unterscheidung der Bestandselemente des Populismus nützlich sein, sei es in Differenz zu anderen Ideologien, ${ }^{72}$

70 Der letzte Parteikongress vor der Krise hat 2002 stattgefunden, obwohl das Statut eine Wiederholung alle drei Jahre vorsieht.

71 Zanatta, Sul nucleo forte di un'ideologia debole.

72 Vgl. Cas Mudde, The Populist Zeitgeist. In: Government \& Opposition, 39 (2004) 4, S. 541-563. 
Aktionsarten ${ }^{73}$ oder Mentalitäten ${ }^{74}$ sei es für eine Identifikation zugehöriger individueller und kollektiver politischer Akteure. ${ }^{75}$

Der Fall der Lega Nord erscheint unter diesen Gesichtspunkten als paradigmatisch. Die Partei hat aus Gründen, die nicht nur auf ihrem plötzlichen Erfolg im politischen System Italiens beruhen, die Aufmerksamkeit italienischer und ausländischer Politikwissenschaftler oder Beobachter auf sich gezogen. Einer dieser Gründe liegt in der Natur der Partei begründet. Die ersten Analysen bezüglich des Erfolges der LN haben vorwiegend die Wichtigkeit der territorialen Forderungen unterstrichen. Dieses Element wurde in den norditalienischen Regionen zum ersten Mal deutlich durch diese Partei artikuliert.

Der Ursprung der Lega wurde also in die Zentrum-Peripherie-Spaltung einer Rokkan-Perspektive miteinbezogen, und die Partei wurde in eine Reihe von ethno-regionalistischen ${ }^{76}$ Konstellationen gestellt, also gewissermaßen als legitimer Vertreter der „questione settentrionale“ („Nordfrage“) verstanden. Zur gleichen Zeit hat jedoch die antipolitische und parteienfeindliche Wende, welche die Partei in ihren Forderungen zwischen Ende der 1980er Jahre und Anfang der 1990er Jahre geprägt hat, eine kontinuierliche und klassische Form von Antiestablishment-Rhetorik hervorgebracht ${ }^{77}$ - all dies im Verlauf einer historischen Krise und Umstrukturierung des italienischen Parteiensystems, die unter dem Einfluss des Falls der Berliner Mauer, der Entdeckung eines weitverbreiteten Systems der Korruption und der unerlaubten Parteienfinanzierung stand. Geschaffen wurde eine populäre und volkstümliche Rhetorik, mit dem Ziel, Feinde des Volkes klar zu identifizieren. Erklärter Zweck war dabei die Strukturierung und Aufwertung einer homogenen Gemeinschaftszugehörigkeit - der des Nordens.

Die Polysemie des Konzeptes Populismus zeigt im Falle der Lega deutlich, welches die Bezugsthemen der Partei sind. Begründet werden kann dies mit der wechselhaften Bedeutung des Begriffes Padania, aber ebenso anhand des Volksbegriffes. Der von der Lega während der oben beschriebenen Phasen ausgedrückte Identitätspopulismus spiegelt den wechselhaften Bezug auf eine Padania wider, deren Grenzen nie wirklich definiert worden sind, und verweist auf die entsprechenden föderalistischen Ziele bzw. Unabhängigkeits- oder Sezessionsstrategien. Diese Polysemie liegt ebenso in einem Appell an das Volk begründet,

73 Vgl. Taguieff, L’illusion populiste.

74 Vgl. Marco Tarchi, Il populismo e la Scienza Politica. Come liberarsi dal complesso di cenerentola. In: Filosofia Politica, 3 (2004), S. 411-432.

75 Über die mehrdimensionalen Eigenschaften des Populismus siehe: Paula Diehl, Die Komplexität des Populismus. Ein Plädoyer für ein mehrdimensionales und graduelles Konzept. In: TD, 8 (2011) 2, S. 273-291.

76 Vgl. Filippo Tronconi, I partiti etnoregionalisti. La politica dell'identità territoriale in Europa occidentale, Bologna 2009.

77 Vgl. Andreas Schedler, Anti-Political-Establishment Parties. In: Party Politics, 2 (1996) 3, S. 291-312; ders. (Hg.), The End of Politics. Explorations into Modern Antipolitics, London 1997. 
der je nach der Dominanz von Identitäts- oder Protestinhalten auf einer Ausnutzung der Unzufriedenheit der Wähler mit einer Klasse von Berufspolitikern basiert. ${ }^{78}$ Die Geschichte der Partei spiegelt also ein ständiges Schwanken zwischen einer Identitäts- und einer Protestdimension des Appells an das Volk, gegenüber dem die LN bemüht ist, sich als Kollektivität zu präsentieren. Aus diesem Grund befindet sich für einige Autoren die Krönung des Verständnis der langen Dauer des leghismo in der Forderung einer Art „zweckdienlichen Einwilligung“ der Padania-Idee auf Basis einer kulturellen, aber hauptsächlich fiskalischen Unabhängigkeit der nördlichen Regionen, unter Ausnutzung der bei den Wählern verbreiteten Ängste bezüglich Themen wie Einwanderung, öffentlicher Sicherheit und den Herausforderungen, die in der islamischen Welt und der Globalisierung gesehen werden.

Diese zuletzt genannten Aspekte kennzeichnen die LN als rechtspopulistische Partei - als Mitglied jener breiteren Familie von Parteien also, die mit ihrem Appell an den Durchschnittsbürger Tugenden beschwören, welche dem professionellen Politiker abgehen. Insgesamt geht es um die Konstruktion einer von Ausländern und finanziellen Unsicherheiten, von europäischer Bürokratie und Korruption bedrohten Kollektivität. Einige dieser Themen sind in der Vergangenheit und auch heute zweifellos noch Teil einer rechtsradikalen kulturellen Identität. Die Besonderheit des vielseitigen, von der Lega ausgedrückten Appells an das Volk, welchen die Partei erfolgreich den wechselhaften Bedingungen eines sich wandelnden politischen Systems angepasst hat, macht sie zu einem typischen Beispiel jener wechselhaften Natur, welche den Populismus kennzeichnet und welche ihn im Gegensatz zu anderen Strukturen oftmals dazu bringt, exogene Erschütterungen zu ertragen. Die heutige Herausforderung der Partei besteht darin, eine Vielzahl endogener Krisenfaktoren zu bewältigen: Vom Austritt der alten Führer hin zur organisatorischen Umstrukturierung, die auf interne Skandale folgte und welche ernsthaft die Glaubwürdigkeit der LN im Volke bedroht.

78 Vgl. Leonard Morlino/Marco Tarchi, The dissatisfied society: the roots of political change in Italy. In: European Journal of Political Research, 30 (1996) 1, S. 41-63. 